логий, в свою очередь, характеризуется высокой динамикой, разработкой все более совершенных приложений для моделирования электронных устройств. Поэтому темой выбрана разработка методического пособия для изучения устройств электроники в вузе в одной из последних версий моделирующей программы Multisim.

Основными частями данного методического пособия являются следующие разделы:

1) исследование арифметико-логического устройства в режиме сложения;

2) исследование арифметико-логического устройства в режиме вычитания;

3) исследование арифметико-логического устройства в режиме умножения;

4) исследование арифметико-логического устройства в режиме деления;

5) исследование арифметико-логического устройства в режиме выполнения логических операций;

6) исследование аналого-цифровых преобразователей;

7) исследование цифро-аналоговых преобразователей.

Разделы указаний состоят из целевой установки выполнения работы, перечня оборудования, используемого при проведении эксперимента, кратких теоретических сведений, из описания порядка проведения и изложения результатов соответствующего эксперимента.

Эта работа, по нашему мнению, может послужить более глубокому и полному изучению теории устройств электроники, что, в свою очередь, обусловит достижение более высоких результатов в области энерго- и ресурсосбережения в машиностроении, электроэнергетике и других областях науки и техники.

\title{
МАТЕМАТИЧЕСКИЙ МЕТОД КОНТРОЛЯ ДОСТОВЕРНОСТИ ИЗМЕРИТЕЛЬНОЙ ИНФОРМАЦИИ О ПОТОКАХ ЭНЕРГЕТИЧЕСКИХ РЕСУРСОВ НА ОСНОВЕ ТЕОРИИ ОЦЕНИВАНИЯ СОСТОЯНИЯ
}

Самойленко B.О., Паздерин А.В. УpФУ,vedmed@e1.ru,pav@daes.ustu.ru

Вопросы энергетической эффективности выходят сегодня на первое место во всех отраслях ТЭК. Известно, что повышение энергоэффективности должно начинаться с создания высокотехнологичной системы измерения и учета энергоресурсов, которая позволяет совершенствовать систему финансовых взаиморасчетов между сторонами. В настоящее время идет активный процесс создания автоматизированных информационно-измерительных систем контроля и учета энергетических ресурсов (АИИС КУЭ).

Основой транспорта большинства видов энергоресурсов является развитая сетевая инфраструктура. Наличие трубопроводов, теплотрасс и электрических сетей как элементов данной инфраструктуры делает схожими нефтегазовую отрасль, теплоэнергетику и электроэнергетику. Как следствие, схожи и математические модели при исследованиях в данных областях, хотя физическая сущность процессов различна. Систему транспорта энергоресурсов можно представить в виде графа, узлами которого являются производители и потреби- 
тели, а ветви являются транспортными магистралями (линии электропередачи, трубопроводы и тепломагистрали).

В статье представлен математический метод контроля достоверности измерений в сети транспорта энергоресурсов, являющийся практическим приложением теории оценивания состояния и задачи энергораспределения [1].

В произвольной сети (рис. 1) с некоторым количеством узлов и ветвей существуют узловые инъекции энергоресурса от производителей (электроэнергия, теплоноситель, поток газа и др.), расход энергоресурса в узлах потребления, перетоки по ветвям (по линиям электропередачи или трубопроводам) и потери в них.

Потери энергоресурса в транспортной ветви определяются спецификой конкретного вида энергоресурса и физической сущностью процесса передачи.

Символом «Х» обозначено наличие и место установки измерительного комплекса энергоресурса. В любой момент времени в сети существует баланс.

Любой измерительный комплекс обладает определенной погрешностью, поэтому баланс в узле по данным измерений не выполняется, а величины потоков не совпадают с расчетными. Задачей энергораспределения является минимизация суммы квадратов относительных ошибок измерений:

$$
F=\sum_{i=1}^{K} r_{i}\left(\frac{E_{i}^{\text {meas }}-E_{i}^{\text {calc }}}{E_{i}^{\text {meas }}}\right)^{2} \rightarrow \text { min },
$$

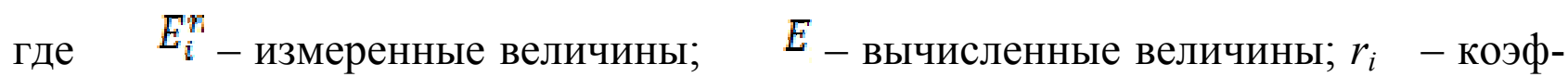
фициенты доверия измерению, обратно пропорциональные квадрату ошибки $\delta_{i}$ измерительного комплекса:

$$
r_{i}=\frac{1}{\left(\delta_{i}\right)^{2}}
$$

Задача энергораспределения - типичная задача нелинейного программирования, решаемая соответствующими методами [2].

В матричном виде систему уравнений для всех измерений можно представить:

где $\mathbf{H}$ - матрица наблюдаемости.

$$
\mathbf{H} \cdot \mathbf{E}_{\mathrm{i}}^{\mathrm{calc}}=\mathbf{E}_{\mathrm{i}}^{\text {meas }},
$$

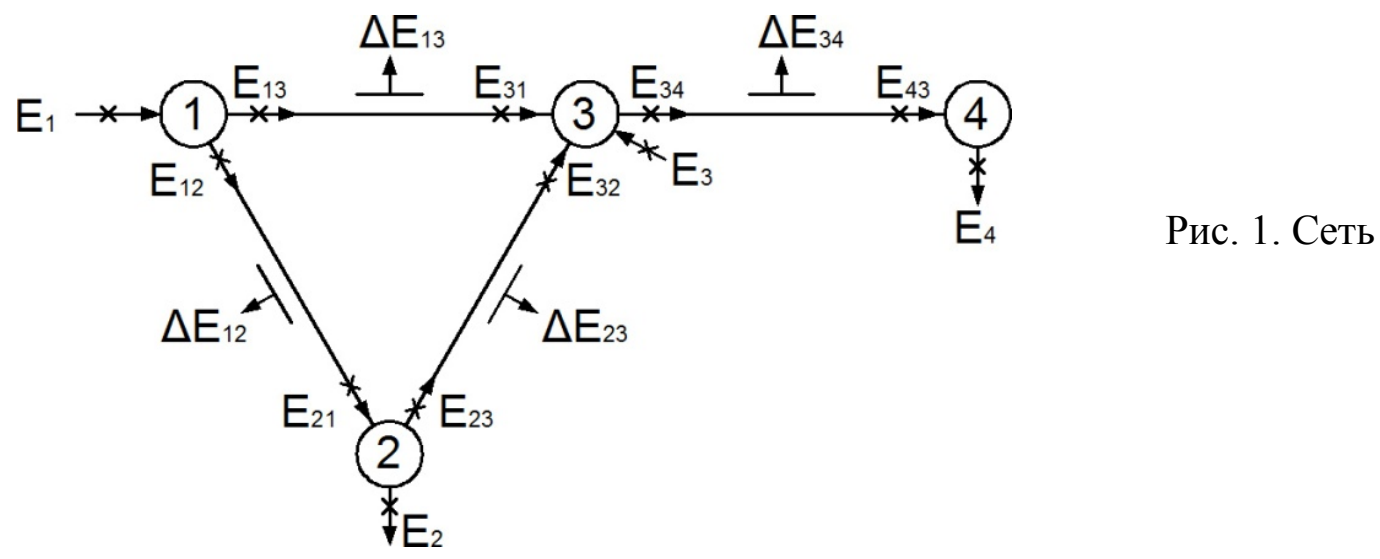


В теории оценивания состояния доказывается, что для обеспечения наблюдаемости схемы число измерительных комплексов должно быть не меньше

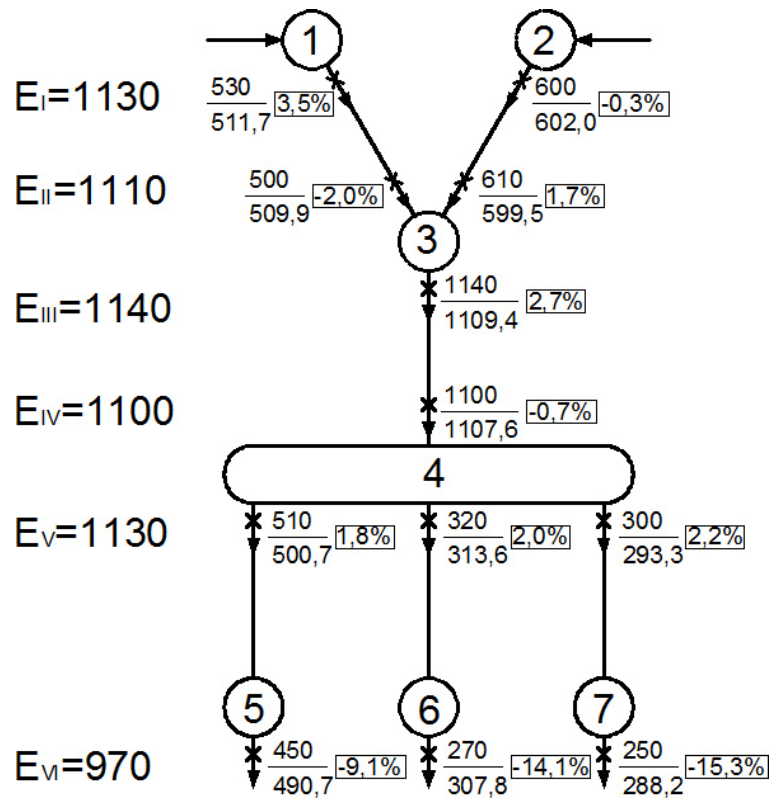

Рис. 2. Тестовая схема числа ветвей в схеме. Данный вывод представляет интерес с точки зрения определения оптимальных мест установки измерительных комплексов в системе [3].

Пример оценивания состояния приведен на рис. 2. Исходная картина измерений по данным соответствующих измерительных комплексов представлена параметрами в числителе. Слева представлены суммарные значения перетока по ветвям на разных уровнях схемы. Ввиду наличия погрешностей измерительных комплексов балансы энергоресурса в схеме не выполняются, а сопоставление втекающих и вытекающих потоков приводит к результатам, противоречащим здравому смыслу и закону сохранения энергии. При решении задачи картина измерений сглаживается, балансы выполняются (числа в знаменателе, указана разница с исходным значением в процентах). Такие данные пригодны для дальнейшего использования. При наличии расчетных данных о технологических потерях разница между фактическими потерями и технологическими будет являться коммерческими потерями [4].

Одной из важнейших проблем является также идентификация недостоверных данных. Метод контроля достоверности позволяет фильтровать плохие данные на стадии сбора информации [5].

Представленный в статье метод позволяет осуществить ряд полезных функций перспективной измерительной системы.

1. Повысить достоверность измерений энергоресурсов (электроэнергия, газ, нефть, теплоноситель), транспорт которых осуществляется с помощью сетевой инфраструктуры.

2. Выбор оптимальных точек установки измерений энергоресурсов на основе теории оценивания состояния.

3. Сглаживание неточных измерений и незначительных несоответствий в показаниях группы измерительных комплексов для получения соответствия.

4. Обнаружение недостоверных данных с возможностью их повторного запроса, а также обнаружение источника недостоверной информации и сигнализация о неисправности в системе. Оценка уровня коммерческих потерь.

Представленный метод может лечь в основу высокотехнологичных систем измерения и учета энергоресурсов нового поколения.

\section{Библиографический список}

1. Solution of energy flow problem using state estimation technique // 35th Annual Conference of IEEE Industrial Electronics Society. Porto. Portugal. 2009. P. 1750-1755. 
2. Pazderin A.V., Plesnyaev E.A. Analysis of the Computation Techniques for Energy Flow Problem Solving // Proceedings IEEE Conference «Computer as a Tool» (EURO 2005). Belgrade. Serbia \& Montenegro. 2005. P. 589-593.

3. Егоров. А.О. Расстановка измерительных комплексов электроэнергии в сетях на основе теории наблюдаемости. Екатеринбург, 2007.

4. Паздерин А.В. Локализация коммерческих потерь электроэнергии на основе решения задачи энергораспределения // Промышленная энергетика. 2004. № 9. С. 6-20.

5. Паздерин А.В. Способы повышения достоверности измерительной информации систем учета электрической энергии // Изв. вузов. Проблемы энергетики. 2004. № 11-12. C. $79-87$.

\title{
ПРИМЕНЕНИЕ ОШИПОВАННЫХ ПОВЕРХНОСТЕЙ В ТЕПЛООБМЕННЫХ АППАРАТАХ
}

\author{
Самоделкин С.И., Краснова Н.П. \\ Самарский государственный технический университет \\ krasnova_pt@rambler.ru
}

Интенсификация теплообмена является одним из основных направлений развития и усовершенствования тепловых агрегатов. Для улучшения характеристик теплоэнергетического оборудования необходимо разрабатывать новые конструкции теплообменных аппаратов: увеличивать эффективность теплообменных поверхностей, применять современные подходы к проектированию теплообменных аппаратов, создавать новые технологии их производства.

Нашли свое распространение различные способы интенсификации теплообмена в конвективных поверхностях: установка оребренных водогрейных труб, нанесение шероховатости или серии углублений, выступы различной формы, шипы и т. д.

Способ интенсификации теплообмена с применением ошипованной поверхности является одним из самых перспективных, поскольку при его реализации существует опережающий рост относительного коэффициента теплоотдачи по сравнению с ростом относительного коэффициента сопротивления. За основу принципа расположения шипов в конвективных поверхностях нагрева теплогенераторов выбраны исследования характера движения потока через пучки водогрейных труб.

Для осуществления эффективного теплообмена наибольшую роль играет способ размещения интенсифицирующих элементов на поверхности, расстояния между ними, геометрические характеристики.

Характеристикой ошиповки, по аналогии с водогрейными трубами, являются поперечный шаг $\mathrm{s}_{1}$ (расстояние между осями шипов в направлении, поперечном потоку жидкости) и продольный шаг $\mathrm{s}_{2}$ (расстояние между осями соседних двух рядов шипов, расположенных один за другим в направлении течения жидкости). Помимо $\mathrm{s}_{1}$ и $\mathrm{s}_{2}$ шипы характеризуются внешним диаметром и количеством рядов шипов по ходу жидкости. Для определенной ошиповки шаги $\mathrm{s}_{1}$ и $\mathrm{s}_{2}$ и диаметр d обычно являются постоянными, не изменяющимися как поперек, так и вдоль течения жидкости.

На кафедре «Промышленная теплоэнергетика» Самарского государственного технического университета проводятся исследования по применению 